\title{
PAPER
}

\section{Multiple sclerosis in Finland: incidence trends and differences in relapsing remitting and primary progressive disease courses}

\author{
M-L Sumelahti, P J Tienari, M Hakama, J Wikström
}

See end of article for authors' affiliations

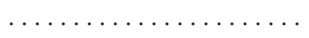

Correspondence to: Dr M-L Sumelahti, School of Public Health, PO Box 607, FIN-33101 Tampere, Finland; memasu@uta.fi

Received 4 February 2002 In revised form 23 September 2002 Accepted 26 September 2002

\begin{abstract}
Objective: To compare the secular trends and geographical differences in the incidence of relapsingremitting (RRMS) and primary progressive multiple sclerosis (PPMS) in Finland, and to draw inferences about aetiological differences between the two forms of the disease.

Methods: New multiple sclerosis cases in southern Uusimaa and the western districts Vaasa and Seinäjoki of Finland in 1979-1993 were verified from hospital records and classified into RRMS and PPMS. Patients met the Poser criteria for definite multiple sclerosis or otherwise satisfied the criteria for PPMS. Disease course was categorised by the same neurologist. Crude and age adjusted incidence in 1979-1993 was estimated.

Results: During 1979-1993 the age adjusted incidence was 5.1 per 100000 person-years in Uusimaa, 5.2 in Vaasa, and 11.6 in Seinäjoki. The rates in Uusimaa remained stable, while a decrease occurred in Vaasa and an increase in Seinäjoki. Between 1979-86 and 1987-93 the incidence of PPMS increased in Seinäjoki from 2.6 to 3.7 per $10^{5}$ and decreased in Vaasa from 1.9 to 0.2 per $10^{5}$; the trends were similar for RRMS.

Conclusions: There are significant differences in secular trends for multiple sclerosis incidence in Finland by geographical area, but these are similar for PPMS and RRMS. The recent changes point to locally acting environmental factors. The parallel incidence trends for RRMS and PPMS suggest similar environmental triggers for the two clinical presentations of multiple sclerosis.
\end{abstract}

7 he clinical spectrum of disease progression in multiple sclerosis is heterogeneous. ${ }^{12}$ In most series about $70-90 \%$ of patients have a relapsing-remitting disease course (RRMS), which may later convert to a progressive phase (secondary progressive multiple sclerosis, SPMS). Approximately $10-30 \%$ of patients have a progressive course from the onset (primary progressive multiple sclerosis, PPMS), ${ }^{2-6}$ with a worse prognosis than for RRMS. ${ }^{7}$ In addition to differences in the natural course, ${ }^{36}$ there is increasing support for pathogenic differences between RRMS and PPMS from neuroimaging ${ }^{8}$ and neuropathological observations. ${ }^{9}$ There is also some evidence that the HLA class II associations may be different. ${ }^{10-12}$ Given these differences, RRMS and PPMS may also be aetiologically distinct, and this should be reflected in the circumstances of their occurrence.

Significant geographical and temporal differences and an increasing occurrence are common observations in multiple sclerosis epidemiology in high risk regions, ${ }^{13}$ and have been reported in Finland. ${ }^{14-19}$ Finland belongs to a high risk region for multiple sclerosis, ${ }^{13}$ with prevalences of from 100 to 200 per 100000 population in different areas. ${ }^{18}$ The western district Seinäjoki has for a long time stood out as a high risk focus of the disease, ${ }^{15}{ }^{16}$ with a prevalence of $200 / 10^{5}$ in 1993 and an incidence of 12 per 100000 person-years in 1979 to $1993 .{ }^{17} 18$ This is among the highest ever reported and is twice as high as the rates in two other areas in Finland, the western region of Vaasa and southern Uusimaa. A familial clustering of multiple sclerosis cases has been observed in Seinäjoki. ${ }^{19}$

The similar high multiple sclerosis incidences of $8 / 10^{5}$ and $10 / 10^{5}$ that were present in Seinäjoki and Vaasa in 1979 to $1983^{17}$ showed a marked change between 1984 and 1993, with a significant increase in Seinäjoki and a decrease in Vaasa. No change was observed in southern Uusimaa. In the present study we compare the occurrence of multiple sclerosis broken down by type (RRMS and PPMS), and analyse the temporal variations in incidence and the clinical and demographic characteristics of the two forms of multiple sclerosis in Finland. This allowed us to investigate whether there are geographical and secular differences in the two disease courses which would point to aetiological differences. Such a hypothesis is feasible because there are several clinical and pathological differences between primary progressive and relapsing remitting multiple sclerosis. ${ }^{3689}$

\section{METHODS}

Demographic data and population

Finland is situated in northern Europe between latitudes $60^{\circ}$ and $70^{\circ}$. The total population was 5.1 million in 1993. The study districts of Uusimaa, Vaasa, and Seinäjoki are shown in fig l. At the end of 1993 the populations were 1277932 , 197042 , and 179079 , respectively, in the three districts. The annual increase in the Finnish population has been $0.4 \%$ from 1979. The population structure was affected by the migration of young adults into Uusimaa. However, the largest age group continues to be $40-49$ years in all districts.

The western district is divided into two health care districts, where two central hospitals provide the neurological services. CSF and evoked potential examinations are equally available, but MRI has only been available in Seinäjoki since 1993. In southern Uusimaa the diagnostic facilities in the 1970s and 1980s were better; however, the situation became equal in the three areas during the 1990s. In Finland, all multiple sclerosis diagnoses from 1960s have been made by a neurologist in the neurological units of university or central hospitals, following the same diagnostic principles.

For the purpose of this study, patients with multiple sclerosis in the health care districts of Uusimaa, Vaasa, and Seinäjoki were ascertained from hospital registers by the diagnoses multiple sclerosis or morbus demyelinans (340 and 341 in the 


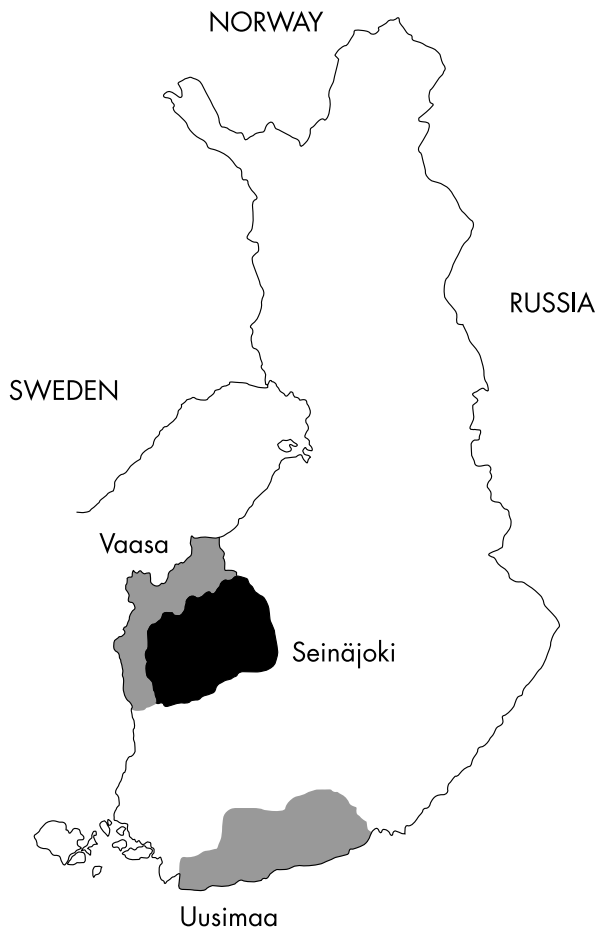

Figure 1 Map of Finland showing the hospital districts under study (Uusimaa in the south and Vaasa and Seinäjoki in the west).

International Classification of Diseases, versions 8 and 9). Patients were re-evaluated to meet the criteria of $\operatorname{Poser}^{20}$ or otherwise they met the criteria for PPMS. ${ }^{21}$ Disease course was classified retrospectively (by M-LS) into RRMS and PPMS groups. ${ }^{22}$ In our study, PPMS was defined as disease that showed steady progression during the first year from the primary symptoms. ${ }^{2521}$ The RRMS group included cases with secondary progression after an initial relapsing-remitting course.

\section{Incidence calculations}

The calculations were based on definite cases of multiple sclerosis. ${ }^{20}$ Incidence was calculated from 1 January 1979 to 31 December 1993 per 100000 person-years in age groups from 10 to 69 years $^{23}$ and in calendar time intervals. The significance of the trend was assessed by classifying the total time period into two subgroups only (1979-1986 and 1987-1993) to reduce random variation caused by small numbers.

As the crude rates may be misleading when comparing two calendar periods, the rates were standardised for age by an indirect method. ${ }^{24}$ The standard rates in each district were those of 1979-1986 in each age group. The resulting standardised rate (SIR) is the ratio of the observed cases in 1987-1993 to those expected if the age specific rates had been those of 1979-1986. Confidence intervals (CI) were calculated assuming that the observed number of cases followed a Poisson distribution.

\section{RESULTS}

In 1979-1993 the total number of cases was 1066, 828 (78\%) in the RRMS group and $238(22 \%)$ in the PPMS group. The number of cases was 736 in Uusimaa, 240 in Seinäjoki, and 90 in Vaasa. The clinical and pathological characteristics of the material are presented in table 1. CSF intrathecal IgG synthesis, which was found in over $90 \%$ of the cases tested, showed no significant differences between PPMS and RRMS groups or between the districts. A lower female to male ratio (F/M) was observed among PPMS cases in each district (1.2-2.0) than among RRMS cases (1.8-2.7). The F/M ratio was especially low for PPMS in Seinäjoki (1.2), as was the ratio for all cases
Table 1 Clinical and pathological characteristics of patients with multiple sclerosis diagnosed in 1979-1993 in Uusimaa, Vaasa, and Seinäjoki districts

\begin{tabular}{|c|c|c|c|}
\hline Characteristic & Uusimaa & Vaasa & Seinäjoki \\
\hline \multicolumn{4}{|c|}{ CSF IgG positivity (\% of tested patients) } \\
\hline All & 91 & 94 & 92 \\
\hline RRMS & 91 & 95 & 91 \\
\hline PPMS & 94 & 88 & 94 \\
\hline \multicolumn{4}{|l|}{ Female to male ratio } \\
\hline All & 2.4 & 2.2 & 1.6 \\
\hline RRMS & 2.7 & 2.3 & 1.8 \\
\hline PPMS & 1.6 & 2.0 & 1.2 \\
\hline \multicolumn{4}{|c|}{ Mean age at diagnosis (years) } \\
\hline All & 35.7 & 39.7 & 39.3 \\
\hline RRMS & 35.4 & 38.7 & 38.4 \\
\hline PPMS & 36.6 & 43.5 & 41.5 \\
\hline \multicolumn{4}{|l|}{ Symptoms at onset (\%) } \\
\hline Motor & 16 & 18 & 30 \\
\hline Pure motor & 13 & 8 & 16 \\
\hline Paraparesis & 3 & 10 & 14 \\
\hline Other & 84 & 82 & 70 \\
\hline Brain stem & 19 & 16 & 21 \\
\hline Sensory & 27 & 39 & 18 \\
\hline Optic neuritis & 18 & 11 & 15 \\
\hline Miscellaneous* & 20 & 16 & 16 \\
\hline \multicolumn{4}{|c|}{ Ratio of motor to other symptoms } \\
\hline RRMS & 0.1 & 0.2 & 0.3 \\
\hline PPMS & 0.5 & 0.4 & 0.9 \\
\hline Total & 0.2 & 0.2 & 0.4 \\
\hline
\end{tabular}

(1.6). Mean age at diagnosis was greater for PPMS (40.5 years) than for RRMS ( 37.5 years), and the age at diagnosis was generally greater in the western districts of Vaasa and Seinäjoki than in Uusimaa. At onset there was a higher proportion of motor symptoms in Seinäjoki (30\%) than in the other districts, and the ratios of motor symptoms to other symptoms by disease course were higher for PPMS in Seinäoki (0.9) and lower for RRMS in Uusimaa (0.1).

The incidences of total multiple sclerosis, RRMS, and PPMS were similar in Uusimaa and Vaasa, but twofold higher in Seinäjoki. The difference in incidence between the RRMS and PPMS groups was somewhat larger (4.6) in Seinäjoki and about the same in Uusimaa and Vaasa (3.1 and 2.8) (table 2).

Trends by five year age groups were stable in Uusimaa but showed an increase in Seinäjoki and a decrease in Vaasa. This trend was similar for RRMS and PPMS (fig 2). Owing to small numbers, especially in Vaasa district $(\mathrm{n}=90)$, the time period 1979-1993 was divided into two periods only (1979-1986 and 1987-1993). The incidence of PPMS increased in Seinäjoki from 2.6 to 3.7 per $10^{5}$ person-years and decreased in Vaasa from 1.9 to 0.2 . The trends were similar for RRMS. The decrease in total and PPMS incidences in Vaasa was statistically significant, but not the decrease in RRMS. Similarly, the increase in Seinäjoki was statistically significant for total multiple sclerosis and PPMS but not for RRMS (table 2).

\section{DISCUSSION}

Over successive studies from the 1960s, Finland has been shown to belong to a high risk region for multiple sclerosis. ${ }^{13}$ The early observation of a high risk in the western district of Seinäjoki ${ }^{14}$ became more evident in the early 1990s, when an incidence of $13 / 10^{5}$ person-years was four times higher than the rates in neighbouring Vaasa $\left(3 / 10^{5}\right)$ and southern Uusimaa $\left(5 / 10^{5}\right) \cdot{ }^{17}$ The rate in Seinäjoki was among the highest reported worldwide. ${ }^{25} 26$ In the present study we show that the secular trend in 1979 to 1993 was increasing in Seinäjoki, decreasing 
Table 2 Number of cases of multiple sclerosis, incidence per 100000 person-years in the age group 10 to 69 years, and standardised incidence ratios by calendar time, district, and disease course, 1979 to 1993

\begin{tabular}{|c|c|c|c|c|c|c|c|c|c|c|c|}
\hline \multirow[b]{3}{*}{ District } & \multirow{3}{*}{$\begin{array}{l}\text { Disease } \\
\text { course }\end{array}$} & \multicolumn{10}{|c|}{ Calendar time } \\
\hline & & \multicolumn{3}{|c|}{1979 to 1986} & \multicolumn{4}{|c|}{1987 to 1993} & \multicolumn{3}{|c|}{1979 to 1993} \\
\hline & & $N$ & I & SIR & $N$ & I & SIR & $\mathrm{Cl}$ & $N$ & 1 & $\mathrm{Cl}$ \\
\hline \multirow{3}{*}{ Uusimaa } & RRMS & 301 & 4.1 & Reference & 290 & 4.2 & 1.0 & 0.9 to 1.2 & 591 & 4.1 & 3.8 to 4.5 \\
\hline & PPMS & 75 & 1.0 & Reference & 70 & 1.0 & 1.0 & 0.8 to 1.3 & 145 & 1.0 & 0.8 to 1.2 \\
\hline & Total & 376 & 5.1 & Reference & 360 & 5.2 & 1.0 & 0.9 to 1.1 & 736 & 5.1 & 4.8 to 5.5 \\
\hline \multirow[t]{3}{*}{ Vaasa } & RRMS & 42 & 4.2 & Reference & 27 & 3.1 & 0.7 & 0.5 to 1.1 & 69 & 4.0 & 3.0 to 4.9 \\
\hline & PPMS & 19 & 1.9 & Reference & 2 & 0.2 & 0.1 & 0.02 to 0.4 & 21 & 1.2 & 0.7 to 1.7 \\
\hline & Total & 61 & 6.0 & Reference & 29 & 3.3 & 0.5 & 0.4 to 0.8 & 90 & 5.2 & 4.1 to 6.3 \\
\hline \multirow[t]{3}{*}{ Seinäjoki } & RRMS & 83 & 6.8 & Reference & 85 & 7.9 & 1.2 & 0.9 to 1.4 & 168 & 8.1 & 6.9 to 9.3 \\
\hline & PPMS & 32 & 2.6 & Reference & 40 & 3.7 & 1.4 & 1.02 to 1.9 & 72 & 3.5 & 2.7 to 4.3 \\
\hline & Total & 115 & 9.4 & Reference & 125 & 11.6 & 1.2 & 1.03 to 1.5 & 240 & 11.6 & 10.1 to 13.1 \\
\hline
\end{tabular}

$\mathrm{Cl}$, confidence interval; I, incidence; $\mathrm{N}$, number of cases; PPMS, primary progressive multiple sclerosis; RRMS, relapsing-remitting multiple sclerosis; SIR, standardised incidence ratio.

in Vaasa, and stable in Uusimaa for the disease as a whole, as well as for the PPMS and RRMS subtypes.

The populations of western and southern districts of Finland differ economically as well as in their migration pattern, immigration being greatest in the urbanised Uusimaa but stable in the more rural populations of Vaasa and Seinäjoki. Owing to the longstanding isolation of Finland and a low migration rate within the country until recently, large areas have remained genetically isolated. The population density in the country as a whole is low and even now the growth of the gene pool is restricted. ${ }^{27}{ }^{28}$ This is shown in the HLA distribution, as allelic differences in HLA can still be found across
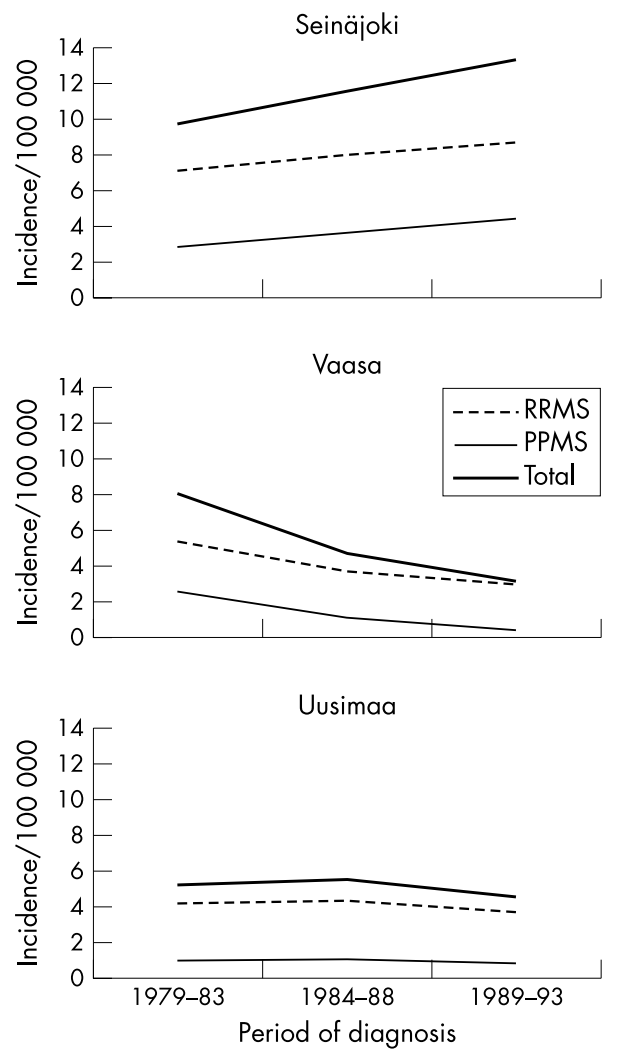

Figure 2 Incidence trends per 100000 person-years in the disease course of all multiple sclerosis cases, relapsing-remitting multiple sclerosis (RRMS), and primary progressive multiple sclerosis (PPMS) in Seinäjoki, Vaasa, and Uusimaa in 1979-1993. the country. Variations in the frequencies of A9, B35, and Cw4 are common in Finland. ${ }^{29}$

Medical practice in Finland is by and large homogeneous. Neurological facilities in the Finnish health care system are provided in the local or central hospitals of each health care district. Thus multiple sclerosis is diagnosed almost exclusively at the neurological units of these hospitals. Populations under study are reasonably large, representing an industrialised population in Uusimaa and rural populations in Vaasa and Seinäjoki. To improve the comparability between the districts, the time point of definite diagnosis was classified retrospectively, and the diagnostic tests were recorded. The diagnosis was mainly clinical in over $80 \%$ of the cases. ${ }^{17}$ Classification of disease course was done by the same neurologist (M-LS) which should reduce potential inter-rater classification bias. ${ }^{42}$ Because of the commonly recognised classification problems in multiple sclerosis, ${ }^{30}{ }^{31}$ we decided to use only two disease course categories, relapsing remitting and primary progressive, the former category including patients who later converted into the secondary progressive form.

Our population sample showing different incidence trends in the three districts provided an opportunity to test the hypothesis that the RRMS and PPMS subtypes could have a different aetiology $y^{32}$ in stable and homogeneous study populations. The fact that trends in the incidence of the two disease subtypes moved in parallel in three districts in which there were diverging trends suggests that the environmental triggers for these two presentations of multiple sclerosis are similar. Our results thus contrast with earlier findings in western Norway, ${ }^{33}$ where the incidence of PPMS remained stable while there was an increasing incidence of RRMS and progressive-relapsing multiple sclerosis during 1953 to 1982. However, these data were based on a much smaller number of patients, and the incidence trends were later shown to be similar for both disease courses. ${ }^{34}$ The fluctuating trends typical in high risk regions for multiple sclerosis were also observed in our study and were shown to be similar for both disease courses. Such fluctuations can most readily be accounted for by environmental exposures.

The age adjusted incidence of PPMS in 1987-1993 was remarkably high in Seinäjoki $\left(3.7 / 10^{5}\right)$ compared with Uusimaa $\left(1.0 / 10^{5}\right)$ and Vaasa $\left(0.2 / 10^{5}\right)$, indicating a long term high risk for PPMS in Seinäjoki. To our knowledge such large regional differences in PPMS occurrence have not been found before within a single population sample. ${ }^{45}$ We do not believe this finding is hampered by diagnostic misclassification ${ }^{6}$ (for example, chronic spastic paraparesis), and this interpretation is supported by the $94 \%$ positive CSF IgG findings among the $96 \%$ of PPMS cases tested in Seinäjoki. The distribution of 
cases in our incidence data-78\% in the RRMS group and 22\% in the PPMS group-follows the common pattern, ${ }^{5}$ as do the demographic features: PPMS is commonly observed among men and is associated with a later age of onset and the likelihood of initial motor symptoms. ${ }^{368}$ Given the recent increase in incidence among men in Seinäjoki, and a lower F/M ratio compared with other districts, ${ }^{17}$ it would be reasonable to suppose that PPMS risk is associated with male sex in Seinäjoki. This was not, however, the case, as the risk for PPMS was high for both sexes (data not shown).

The causes of a locally high and increasing risk of multiple sclerosis, an overall high male risk, ${ }^{17}$ a high risk for both disease types in Seinäjoki, and a contrasting trend in Vaasa remain unexplained. In spite of the language difference between the partly Swedish speaking Vaasa and Finnish speaking Seinäjoki, the genetic background is similar. ${ }^{27}$ In the multiple sclerosis population, cases have been characterised for HLA in the district of Seinäjoki and have been found to have increased frequencies for B7, B12, and DR2 among both patients and their healthy relatives. ${ }^{35}$ Families were later studied for the myelin basic protein (MBP) gene on chromosome 18 , a candidate gene in multiple sclerosis. Genetic linkage and association analyses suggested that a genetic predisposition to multiple sclerosis is closely linked to the MBP gene in this population. ${ }^{36}$ In spite of these observations, our findings of a sustained increase in the incidence of the disease point to environmental factors, as genetic change in populations is slow. The environmental causes are generally suspected to be of viral origin ${ }^{37}$ but remain largely unknown at present.

The large regional differences in the incidence of multiple sclerosis in Finland in 1989-1993 result from diverging trends in incidence that are largely parallel for both types of disease course. In the originally high risk area of Seinäjoki the incidence of both types of course was still increasing from the late 1970s to the early 1990s. In Uusimaa, the incidence of both types has remained stable. In Vaasa, an intermediate incidence in 1979-1983 decreased to a low level for both PPMS and RRMS. This finding is of aetiological importance, as diagnostic practices have remained the same. The sharply diverging incidence trends in Seinäjoki and Vaasa point towards recent changes in environmental factors, and the parallel incidence trends for PPMS and RRMS in all three districts suggest that there are similar environmental triggers for both clinical types of multiple sclerosis.

\section{ACKNOWLEDGEMENTS}

This study was supported by the Sigrid Juselius Foundation, Helsinki University Central Hospital, the University of Tampere, and the Ministry of Education in Finland through a doctoral student position in Doctoral Programmes in Public Health (to M-LS).

\section{Authors' affiliations}

M-L Sumelahti, M Hakama, School of Public Health, University of Tampere, Tampere, Finland

J Tienari, J Wikström, Department of Neurology, University of Helsinki Hospital, Helsinki, Finland

Competing interests: none declared

\section{REFERENCES}

1 Charcot JM. Lectures on the disease of the nervous system. Translated by Sigerson G. London: The New Sydenham Society, 1877:157-222.

2 McAlpine D, Compston ND, Lumsden CE. Multiple sclerosis. Edinburgh Livingstone, 1955.

3 Confavreux C, Aimard G, Devic M. Course and prognosis of multiple sclerosis assessed by the computerized data processing of 349 patients. Brain 1980;103:281-300.

4 Minderhoud J, van der Hoeven J, Prange A. Course and prognosis of chronic progressive multiple sclerosis. Results of an epidemiological study. Acta Neurol Scand 1988;78:10-15.
5 Weinshenker BG, Bass B, Rice GP, et al. The natural history of multiple sclerosis: a geographically based study. I. Clinical course and disability. Brain 1989;112:133-46.

6 Thompson A, Polman C, Miller D, et al. Primary progressive multiple sclerosis. Brain 1997:120:1085-96.

7 Phadke J. Clinical aspects of multiple sclerosis in north-east Scotland with particular reference to its course and prognosis. Brain 1990;1 13:1597-628

8 Thompson A, Kermode A, MacManus D, et al. Patterns of disease activity in multiple sclerosis: clinical and magnetic resonance study. BM 1990;300:631-4

9 Revesz T, Kidd D, Thompson A, et al. A comparison of the pathology of primary and secondary progressive multiple sclerosis. Brain 1994; 11 17:759-65.

10 Olerup 0 , Hillert J, Fredrikson S, et al. Primarily chronic progressive and relapsing/remitting multiple sclerosis: two immunogenetically distinct disease entities. Proc Natl Acad Sci USA 1989;86:7113-17.

11 Masterman T, Ligers A, Olsson T, et al. HLA-DR 15 is associated with lower age at onset in multiple sclerosis. Ann Neurol 2000;48:21 1-19.

12 Weatherby S, Thomson W, Pepper L, et al. HLA-DRB 1 and disease outcome in multiple sclerosis. J Neurol 2001;248:304-10.

13 Kurtzke J. Geography in multiple sclerosis. J Neurol 1977:215:1-26.

14 Rinne U, Panelius M, Kivalo $E$, et al. Distribution of multiple sclerosis in Finland with special reference to some geological factors. Acta Neurol Scand 1966;:42:385-99.

15 Wikström J, Palo J. Studies on the clustering of multiple sclerosis in Finland. I. Comparison between the domiciles and places of birth in selected subpopulations. Acta Neurol Scand 1975;51:85-98.

16 Kinnunen E, Wikström J, Porras J, et al. The epidemiology of multiple sclerosis in Finland: Increase of prevalence and stability of foci in high-risk areas. Acta Neurol Scand 1983;67:255-62.

17 Sumelahti M-L, Tienari P, Wikström J, et al. Regional and temporal variation in the incidence of multiple sclerosis in Finland in 1979-1993. Neuroepidemiology 2000; 19:67-75.

18 Sumelahti M-L, Tienari P, Wikström J, et al. Increasing prevalence of multiple sclerosis in Finland. Acta Neurol Scand 2001;103:1-6.

19 Wikström J. Studies on clustering of multiple sclerosis in Finland. II. Microepidemiology in one high-risk county with special reference to familial cases. Acta Neurol Scand 1975;51:173-83.

20 Poser CM, Paty DW, Scheinberg L, et al. New diagnostic criteria for multiple sclerosis: guidelines for research protocols. Ann Neurol 1983:13:227-31.

21 Lublin FD, Reingold SC. Defining the clinical course of multiple sclerosis: results of an international survey. Neurology 1996;46:907-1 1 .

22 Kremenchutzky M, Cottrell D, Rice G, et al. The natural history of multiple sclerosis: a geographically based study 7 . Progressive-relapsing and relapsing-progressive multiple sclerosis: a re-evaluation. Brain 1999; 122:1941-9.

23 Noseworthy J, Paty D, Wonnacot T, et al. Multiple sclerosis after age 50. Neurology 1983;33:1537-44.

24 Armitage P, Berry G. Standardization. In: Armitage P, Berry G, eds Statistical methods in medical research. Cambridge: Blackwell Science, 1994:436-43.

25 Robertson N, Compston DAS. Surveying multiple sclerosis in the United Kingdom. J Neurol Neurosurg Psychiatry 1995;58:2-6.

26 Warren S, Warren K. Prevalence, incidence and characteristics of multiple sclerosis in Westlock County, Alberta, Canada. Neurology 1993;43: 1760-963.

27 Nevanlinna HR. Genetic markers in Finland. Haematologia 1980;13:65-74.

28 de la Chapelle A. Disease gene mapping in isolated human populations: the example of Finland. J Med Genet 1993;30:857-65.

29 Sirén $M$, Sareneva $H$, Lokki $M$, et al. Unique HLA antigen frequencies in the Finnish population. Tissue Antigens 1996:48:703-7.

30 Khurram B, Whitaker N. Clinical and laboratory features of primary progressive and secondary progressive MS. Neurology 1999;53:765-71

31 McDonnell G, Hawkins S. Clinical study of primary progressive multiple sclerosis in Northern Ireland, UK. J Neurol Neurosurg Psychiatry 1998;64:451-4.

32 Larsen J, Kvale G, Riise T, et al. Multiple sclerosis - more than one disease. Acta Neurol Scand 1985;72:145-50.

33 Larsen JP, Aarli $\mathrm{H}$, Nyland $\mathrm{H}$, et al. Western Norway, a high-risk area for multiple sclerosis: a prevalence/incidence study in the county of Hordaland. Neurology 1984;34:1202-7.

34 Grønning M, Riise T, Kvale G, et al. Incidence of multiple sclerosis in Hordaland, Western Norway: a fluctuating pattern. Neuroepidemiology $1991 ; 10: 53-61$.

35 Kinnunen E, Koskimies S, Lagerstedt A, et al. Histocompatibility antigens in familial multiple sclerosis in a high-risk area of the disease. J Neurol Sci 1984; 65: 147-55

36 Tienari PJ, Wikström J, Sajantila A, et al. Genetic susceptibility to multiple sclerosis linked to myelin basic protein gene. Lancet 1992; 340:987-91.

37 Challoner P, Smith K, Parker J, et al. Plaque-associated expression of human herpesvirus 6 in multiple sclerosis. Proc Natl Acad Sci USA 1995 92:7440-4. 